Pacific Journal of Mathematics

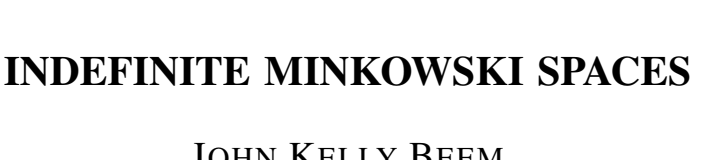




\title{
INDEFINITE MINKOWSKI SPACES
}

\author{
JOHN K. BEEM
}

The purpose of this article is to characterize Minkowski general $G$-spaces. The unit sphere $K$ is shown to have at most four components.

Assume the space $R$ is not reducible. If $K$ has one component, $R$ is an ordinary Minkowski $G$-space. If $K$ has two components they are quadrics and $R$ is nearly pseudoeuclidean. When $K$ has three components, one is a quadric and the other two are strictly convex. The unit sphere has four components only in dimension two.

The axioms of a general $G$-space have been given in [4] and the interesting two dimensional spaces have been investigated in [1]. We will denote the indefinite distance from $x$ to $y$ by $x y$. We refer to $x y$ as a metric even though it is not in general a true metric.

Definition 1.1. The general $G$-space $R$ is called a Minkowski space if $R$ is the real $n$-dimensional affine space $A^{n}$, the family of Arcs $A$ consists of the affine segments and $w=(1 / 2)(x+y)$ implies $w x=$ $w y=(1 / 2) x y$.

If $L^{r}$ is an $r$-dimensional flat in $R$, then $L^{r}$ is an $r$-dimensional Minkowski space with the induced distance.

Let $e(x, y)$ be an associated euclidean metrization of $A^{n}$. Then for each line $L$ in $R$ there is a number $\phi(L)$ such that $x y=\phi(L) e(x, y)$ for all $x, y \in L$. If $\phi(L)=0$, we call $L$ a null line. The number $\phi(L)$ depends continuously on $L$ and $\phi(L)=\phi\left(L_{1}\right)$ if $L_{1}$ is parallel to $L$, see [1]. It follows that the affine translations preserve the distance $x y$.

Let $z$ always denote the origin in $A^{n}$. We call $C=\{x \mid x z=0\}$ the light cone and $K=\{x \mid x z=1\}$ the unit sphere. If $K$ is given the distance $x y$ is uniquely determined.

For $x \neq y$ let $L(x, y)$ denote the line through $x$ and $y$ and let $\alpha(x, y)$ denote the affine segment from $x$ to $y$. When $S \subset A^{n}$ define $-S=\{x \mid-x \in S\}$. If $S=-S$ the set $S$ is called symmetric about $z$ or simply symmetric. The sets $C$ and $K$ are symmetric.

Two general $G$-spaces $R_{1}$ and $R_{2}$ are said to be topologically isometric if there exists a topological map of $R_{1}$ onto $R_{2}$ that preserves the indefinite distance $x y$.

It is easily seen that if $R_{1}$ and $R_{2}$ are Minkowski spaces defined on $A^{n}$ with unit spheres $K$ and $K^{*}$ respectively, then $R_{1}$ and $R_{2}$ are 
topologically isometric if and only if there is an affinity mapping $K$ onto $K^{*}$.

2. Two dimensional spaces. If $R$ is $A^{2}$, then by [4, p. 241] one of the following must hold: (1) no null lines exist in $R,(2)$ there is exactly one null line through each point of $R$, (3) there are exactly two null lines through each point of $R$, or (4) all lines in $R$ are null.

In case (1) we call $R$ a spacelike plane. By [4, p. 239], a spacelike plane is an ordinary Minkowski $G$-space with unit sphere a strictly convex closed curve.

In case (2) we call $R$ a neutral plane. A neutral plane is topologically isometric to the $(s, t)$ plane with distance from $\left(s_{1}, t_{1}\right)$ to $\left(s_{2}, t_{2}\right)$ given by $\left|t_{1}-t_{2}\right|$.

When $R$ has exactly two null lines through each point it is called a doubly timelike (Minkowski) plane, see [1]. The unit sphere has four components each of which is strictly convex and not compact.

If all lines in $R$ are null, we call $R$ a null plane.

3. Reducible spaces, Let $R$ be an $n$-dimensional Minkowski space. Then $R$ is reducible to $R^{r} \times N^{n-r}$ for $r<n$, provided affine coordinates $x_{1}, x_{2}, \cdots, x_{n}$ may be chosen such that

(1) $R^{r}$ is given by $x_{r+1}=x_{r+2}=\cdots=x_{n}=0$ and $N^{n-r}$ is given by $x_{1}=\cdots=x_{r}=0$.

(2) The projection of $R$ onto $R^{r}$ preserves the metric $x y$.

The maximum possible value of $n-r$ is called the index of reducibility of $R$. A null plane has index 2 and a neutral plane index 1 . Spacelike and doubly timelike planes are not reducible.

Nonreducible spaces often contain reducible subspaces. In the three dimensional Lorentz space any plane tangent to the light cone is neutral and hence reducible.

Given a line $N$ the parallel to $N$ through $x$ will always be denoted by $N_{x}$.

Definition 3.1. A line $N$ through $z$ is called a line of reduction of $R$ if $x \in K$ implies $N_{x} \subset K$.

Lemma 3.2. The space $R$ is reducible if and only if $R$ has a line of reduction.

Proof. If $N$ is a line of reduction of $R$ and $L^{n-1}$ is a hyperplane with $L^{n-1} \cap N=z$, the projection of $R$ onto $L^{n-1}$ along parallels to $N$ preserves the metric.

On the other hand if $R$ is reducible to $R^{r} \times N^{n-r}$ any line $N$ through $z$ and in $N^{n-r}$ is a line of reduction of $R$. 
4. The $r$-flat topology. If $\left\{M_{m}\right\}$ is a sequence of closed subsets of $R$, we say $M_{m}$ converges to the closed set $M$ if $\lim M_{m}=M$ in the sense of Hausdorff's closed limit, see [2]. This limit induces a topology on the closed subsets of $R$. If $L^{r}$ is an $r$-flat and $W\left(L^{r}\right)$ is a neighborhood of $L^{r}$ in this topology, let $W_{r}\left(L^{r}\right)$ denote the $r$-flats in $W\left(L^{r}\right)$.

Lemma 4.1. Let $\left\{L_{m}^{2}\right\}$ be a sequence of doubly timelike planes, each containing $z$, such that $\left\{L_{m}^{2}\right\}$ converges to the two flat $L^{2}$. Assume $x_{i}^{m} \in K \cap L_{m}^{2}$ and $x_{i}^{m} \rightarrow x_{i}$ for $i=1,2$.

(1) Let $L^{2}$ be doubly timelike and let $x_{1}, x_{2}$ lie on the same component [opposed components] of $K$. Then for sufficiently large $m$ the points $x_{1}^{m}$ and $x_{2}^{m}$ always lie on the same component [opposed components] of $K \cap L_{i n}^{2}$.

(2) If $L^{2}$ is neutral, then for sufficiently large $m$ the points $x_{1}^{m}$ and $x_{2}^{m}$ are always on the same or else always on opposed components of $K \cap L_{m}^{2}$.

Proof. The proofs are similar and consequently we only consider statement (2) in which $L^{2}$ is neutral.

Without loss of generality assume $x_{1}$ and $x_{2}$ are on the same component of $K \cap L^{2}$ since if $x_{1}^{m} \rightarrow x_{1}$ then $-x_{1}^{m} \rightarrow-x_{1}$.

If $y \in \alpha\left(x_{1}, x_{2}\right)$ then $y \in K$ and $z y=1$. Therefore, there exists an open set $V$ containing the set $\alpha\left(x_{1}, x_{2}\right)$ such that all $p \in V$ have $z p>0$. For sufficiently large $m$ all points of $\alpha\left(x_{1}^{m}, x_{2}^{m}\right)$ lie in $V$ and have positive distance from $z$. It follows that $x_{1}^{m}$ and $x_{2}^{m}$ lie on the same component of $K \cap L_{m}^{2}$ for large $m$.

The components of $K$ are arcwise connected since they are connected and locally arcwise connected.

LEMmA 4.2. Let $x_{1}$ and $x_{2}$ lie on the same component of $K$ and let $L^{2}$ be a two flat containing $z, x_{1}$ and $x_{2}$. If $S_{1}$ and $S_{2}$ are the components of $K \cap L^{2}$ containing $x_{1}$ and $x_{2}$ respectively then either $S_{1}=S_{2}$ or else $S_{1}=-S_{2}$.

Proof. Let $x(t)$ for $0 \leqq t \leqq 1$ be a curve on $K$ connecting $x_{1}$ and $x_{2}$ with $x(0)=x_{1}$ and $x(1)=x_{2}$.

Call the two flat $L^{2}(t)$ admissible if $z, x_{1}, x(t) \in L^{2}(t)$ and $K \cap L^{2}(t)$ has components $S_{1}$ and $S(t)$ containing $x_{1}$ and $x(t)$ respectively such that either $S_{1}=S(t)$ or else $S_{1}=-S(t)$. For sufficiently small $t$ there must exist admissible $L^{2}(t)$. Set $M=\{t \in[0,1] \mid$ there exists an admissible $\left.L^{2}(t)\right\}$.

We now show $M$ is closed. If $\left\{L^{2}\left(t_{m}\right)\right\}$ is a sequence of admissible planes and $t_{m} \rightarrow t_{0}$, then there is a convergent subsequence $\left\{L^{2}\left(t_{k}\right)\right\} \subset$ $\left\{L^{2}\left(t_{m}\right)\right\}$ such that $L^{2}\left(t_{k}\right) \rightarrow L_{0}^{2}$. Clearly $z, x_{1}, x\left(t_{0}\right) \in L^{2}\left(t_{0}\right)$. Statement (1) 
of Lemma 4.1 implies $L_{0}^{2}$ cannot be doubly timelike with $x_{1}$ and $x\left(t_{0}\right)$ neither on the same nor on opposed components of $K \cap L_{0}^{2}$. Therefore, $t_{0} \in M$.

To show $M$ is open let $\tau \in M$ and $L^{2}(\tau)$ be admissible. If $L^{2}(\tau)$ is spacelike there must exist a neighborhood $W_{2}\left(L^{2}\right)$ containing only spacelike planes. But this implies the existence of a neighborhood $U(\tau)$ of the number $\tau$ with $U(\tau) \subset M$. If $L^{2}(\tau)$ is a doubly timelike plane statement (1) of Lemma 4.1 implies the existence of a neighborhood $U(\tau) \subset M$. In case $L^{2}(\tau)$ is a neutral plane first construct a neighborhood $W_{2}\left(L^{2}(\tau)\right)$ in which no null planes exist. If only spacelike and neutral planes exist in $W_{2}\left(L^{2}(\tau)\right)$ there is nothing to show. If there is a sequence of doubly timelike planes $L^{2}\left(t_{m}\right)$ converging to $L^{2}(\tau)$, statement (2) of Lemma 4.1 guarantees that for large $m$ the planes $L^{2}\left(t_{m}\right)$ are admissible. It follows that there is a neighborhood $U(\tau) \subset M$. Therefore, $M$ is open as well as closed. Since $M \neq \phi, M=$ $[0,1]$ and the lemma is established.

THEOREM 4.3. Let $K_{1}$ and $K_{2}$ be distinct components of $K$ that are opposed (i.e., $K_{2}=-K_{1}$ ). Then $K_{1}$ and $K_{2}$ are convex hypersurfaces.

Proof. Let $K_{1}^{0}=\left\{y \mid \alpha(z, y) \cap K_{1} \neq \phi\right\}$. Then $K_{1}^{0}$ has boundary $K_{1}$ and $y \in K_{1}^{0}$ implies $z y \geqq 1$. If $y_{1}, y_{2} \in K_{1}^{0}$ let $L^{2}$ be a two flat through $z, y_{1}$ and $y_{2}$. Then $L^{2}$ must either be neutral or doubly timelike. In either case $\alpha\left(y_{1}, y_{2}\right) \subset K_{1}^{0}$ if $y_{1}$ and $y_{2}$ lie on the same component of $K_{1} \cap L^{2}$. Clearly $y_{1}$ and $y_{2}$ lie on the same component for $L^{2}$ neutral. If $L^{2}$ is doubly timelike, then $K_{1} \neq K_{2}$ and Lemma 4.2 imply $y_{1}$ and $y_{2}$ lie on the same component of $K_{1} \cap L^{2}$. It follows that $K_{1}^{0}$ is convex and that its boundary $K_{1}$ is a convex hypersurface. In the same fashion one may show $K_{2}$ is a convex hypersurface.

Lemma 4.4. Let $K$ have a component $K_{1}$ that is symmetric about z. Then for each $x \in K_{1}$ there is a two flat $L^{2}$ through $z$ and $x$ that is spacelike.

Proof. Assume the statement is false. Any two flat containing $L(z, x)$ is then either neutral or doubly timelike. Orient $L(z, x)$ to get $L^{+}(z, x)$. If $L_{1}$ is a line parallel to $L^{+}(z, x)$, orient $L_{1}^{+}$in the same direction. This gives an ordering $<$ on each line parallel to $L(z, x)$.

Let $x(t)$ for $0 \leqq t \leqq 1$ be a curve on $K_{1}$ with $x(0)=x, x(1)=-x$ and $x(t) \notin L(x,-x)$ for $0<t<1$. Let $L^{+}(t)$ be the oriented line containing $x(t)$ and parallel to $L^{+}(z, x)$. The line $L^{+}(t)$ is never a null line.

In the ordering $<$ along $L^{+}(t)$ let $p(t)$ be the first element in $\left\{y \mid y \in L^{+}(t)\right.$ and $\left.z y=0\right\}$. Let $f(t)$ be the signed euclidean distance from $x(t)$ to $p(t)$ where $f(t)<0$ if $x(t)<p(t)$. If $z<x$ then $f(0)<0$ 
and $f(1)>0$.

The function $f(t)$ is continuous at 0 and 1 since $p(t) \rightarrow z$ for $t \rightarrow 0$ and $t \rightarrow 1$. To show $f(t)$ is continuous on $(0,1)$ let $0<t_{0}<1$ and $t_{m} \rightarrow t_{0}$. For $0<t<1$ let $L^{2}(t)$ denote the unique plane containing $L^{+}(t)$ and $z$. Clearly if $L\left(t_{0}\right)$ is neutral we have $L\left(z, p\left(t_{m}\right)\right) \rightarrow L\left(z, p\left(t_{0}\right)\right)$. If $L^{2}\left(t_{0}\right)$ is doubly timelike, one can show using (1) of Lemma 4.1 that $L\left(z, p\left(t_{m}\right)\right) \rightarrow L\left(z, p\left(t_{0}\right)\right)$. In either case $p\left(t_{m}\right) \rightarrow p\left(t_{0}\right)$ and $f(t)$ is continuous. But then $f(\tau)=0$ for some $0<\tau<1$ which implies $x(\tau)=p(\tau)$. This is impossible since $z x(\tau)=1$ and $z p(\tau)=0$.

5. Three dimensional spaces. In this section we only consider three dimensional Minkowski spaces.

Lemma 5.1. Let $K$ have three components $K_{1}, K_{2}$ and $K_{3}$ with $K_{3}=-K_{3}$. Then $K_{1}=-K_{2}$ and $K_{1}$ (hence also $K_{2}$ ) is strictly convex.

Proof. By Lemma 4.4 there is a two flat $L^{2}$ through $z$ that is spacelike with $L^{2} \cap K_{3} \neq \phi$. This flat separates $A^{3}$ and does not intersect $K_{2}$. Hence $K_{2} \neq-K_{2}$. Consequently, $K_{1}=-K_{2}$.

To see that $K_{1}$ is strictly convex let $x, y \in K_{1}$. If $L_{0}^{2}$ is a two flat through $x, y$ and $z$ it must be doubly timelike since $L_{0}^{2} \cap L^{2} \neq \phi$. Then $L_{0}^{2} \cap K_{1}$ is a strictly convex curve. It follows that $u \in \alpha(x, y)-x-y$ implies $z u>1$. Therefore, $K_{1}$ must be strictly convex.

If $K_{i}$ is a component of $K$ then so is $-K_{i}$. Consequently, if $K$ has exactly three components there is always one, say $K_{3}$, that is symmetric about $z$.

Extend $A^{3}$ to the real three dimensional projective space $P^{3}$ by adding a plane $L_{\infty}^{2}$ at $\infty$. The projective lines that the light cone $C$ determine intersect $L_{\infty}^{2}$ in a curve $C_{\infty}$. Let $K$ have exactly three components. Since spacelike planes exist in this case, there is a line $L_{0} \subset L_{\infty}^{2}$ with $L_{0} \cap C_{\infty}=\phi$. The set $L_{\infty}^{2}-L_{0}$ is an affine plane with $L_{0}$ the line at $\infty$.

Let $p, q \in C_{\infty}$ with $p \neq q$. Let $L^{2}$ be two flat in $P^{3}$ that contains $z, p, q$. Then $L^{2} \cap A^{3}$ cannot be a null plane, since if it were it would separate $A^{3}$ and $K_{3}$ could not be symmetric. Consequently, $L^{2} \cap A^{3}$ must be a doubly timelike plane.

It follows that $L^{2} \cap\left(L_{\infty}^{2}-L_{0}\right)$ is an affine line in $L_{\infty}^{2}-L_{0}$ that intersects $C_{\infty}$ in only the two points $p$ and $q$. But $C_{\infty}$ is a closed curve. Hence, $C_{\infty}$ is a strictly convex curve in $L_{\infty}^{2}-L_{0}$.

Theorem 5.2. Let $\operatorname{dim} R=3$. If $K$ has three components $K_{1}, K_{2}$ and $K_{3}$ with $K_{3}=-K_{3}$, then $K_{3}$ is a hyperboloid of one sheet.

Proof. Let $u \in L_{\infty}^{2}-L_{0}$ and let $u$ be exterior to the convex set 
in $L_{\infty}^{2}-L_{0}$ whose boundary is $C_{\infty}$. Then there are lines $L_{1}$ and $L_{2}$ through $u$ that are supporting lines of $C_{\infty}$. Let $L_{i}^{2}$ be the projective plane containing $z$ and $L_{i}$ for $i=1,2$. Then $L_{i}^{2} \cap C_{\infty}$ is a single point and hence $L_{i}^{2} \cap A^{3}$ is a neutral plane.

The set $L_{i}^{2} \cap A^{3} \cap K$ consists of two parallel lines which must be on $K_{3}$ since $K_{1}$ and $K_{2}$ are strictly convex. For any $q \in K_{3}$ let $u=$ $L(z, q) \cap L_{\infty}^{2}$ and without loss of generality assume $u \notin L_{0}$. Then $u$ must be exterior to $C_{\infty}$. By the above arguments there must be two straight lines on $K_{3}$ through $q$. By [5, p. 272] the set $K_{3}$ is a hyperboloid of one sheet.

Notice that the above theorem gives the additional information that $C$ is elliptic and $C_{\infty}$ is an ellipse in $L_{\infty}^{2}-L_{0}$.

LEMMA 5.3. $K$ can have at most four components. If $K$ does have four components, $R$ is reducible and no component of $K$ is symmetric about $z$.

Proof. Let $K_{1}$ be a component of $K$. Assume $K_{1}=-K_{1}$, then there is a spacelike plane $L_{0}^{2}$ through $z$ with $L_{0}^{2} \cap K_{1} \neq \phi$. Take $K_{2} \neq K_{1}$ and $x \in K_{2}$. Let $L^{2}(\theta)$ be a two flat containing $L(z, x)$ that revolves continuously in $\theta$ and sweeps out $A^{3}$ for $0 \leqq \theta \leqq \pi$. Each $L^{2}(\theta)$ intersects $L_{0}^{2}$ in a line through $z$ so that $L^{2}(\theta) \cap K_{1} \neq \phi$ for all $\theta$. Therefore, each $L^{2}(\theta)$ is doubly timelike and intersects $K$ in four components. Two of these components lie on $K_{1}$, and the other two are subsets of $K_{2}$ and $-K_{2}$. Since this holds for all $\theta \in[0, \pi], K$ can have at most three components. Therefore, $K_{1} \neq-K_{1}$ if $K$ has four components.

By the above, it must be possible to find at least two components $K_{1}$ and $K_{2}$ of $K$ with $K_{1} \neq-K_{1}, K_{2} \neq-K_{2}$ and $K_{1} \neq-K_{2}$. Set $K_{3}=$ $-K_{1}$ and $K_{4}=-K_{2}$. Let $y \in K_{1}$ and let $L^{2}(\psi)$ be a two flat through $L(z, y)$ that sweeps out $A^{3}$ continuously for $0 \leqq \psi \leqq \pi$. It can be assumed without loss of generality that $L^{2}(0) \cap K_{2} \neq \phi$. Therefore, let $x_{2}$ belong to $L^{2}(0) \cap K_{2}$. $\quad L^{2}(\psi)$ cannot be doubly timelike for all $\psi$ or else $x_{2}$ and $-x_{2}$ would be on the same component of $K$. Therefore, there is a first $\psi_{0}$ with $L^{2}\left(\psi_{0}\right)$ neutral. Let $N \subset L^{2}\left(\psi_{0}\right)$ be the null line through $z$. Claim $N$ is a line of reduction of $R$.

It is clear that if $x \in K_{1} \cup K_{3}$ then $N_{x} \subset K_{1} \cup K_{3}$ since these are convex surfaces and $N_{y} \subset K_{1}$ as well as $N_{-y} \subset K_{3}$. For $x \in K_{2} \cup K_{4}$ consider the following argument. Let $L^{2}(\gamma)$ be a plane through $L\left(z, x_{2}\right)$ sweeping out $A^{3}$ continuously for $0 \leqq \gamma \leqq \pi$ with $y \in L^{2}(0)$. By the same reasoning as before, there is a first $\gamma_{0}$ with $L^{2}\left(\gamma_{0}\right)$ neutral. The above $N$ must be in $L^{2}\left(\gamma_{0}\right)$ since $N_{y} \subset K_{1}$ and $K_{1}$ is not flat. This implies $N_{x} \subset K_{2} \cup K_{4}$ whenever $x \in K_{2} \cup K_{4}$.

It is now possible to show $K$ has at most, four components. If $L_{1}^{2}$ is a two flat containing the above $N$ either $L_{1}^{2}$ is neutral or null. 
If it is null, it intersects $L^{2}(\gamma)$ for $\gamma=0$ in a null line. If it is neutral, it intersects either $K_{1}$ and $K_{3}$ or else $K_{2}$ and $K_{4}$. In any case it cannot contain a point of $K$ not on $K_{1} \cup K_{2} \cup K_{3} \cup K_{4}$.

An immediate consequence is that if $K$ has four components $R=$ $R^{2} \times N^{\prime}$ where $R^{2}$ is a doubly timelike plane.

Consider now the case of $K$ having one component. If $R$ has no null lines, then by [4, p. 239] it is a Minkowski $G$-space and $K$ must be strictly convex.

LEMMA 5.4. Let $K$ have one component and not be strictly convex. Then $K$ is a cylinder and $R=R^{2} \times N^{1}$ where $R^{2}$ is a spacelike plane.

Proof. Let $K$ contain a segment $\alpha$ and consider the two flat $L_{0}^{2}$ through $z$ and $\alpha$. $L_{0}^{2}$ must be neutral, hence the line containing $\alpha$ must lie on $K$. Let $N$ be the null line in $L_{0}^{2}$ through $z$. Since $K$ has only one component, there is a spacelike plane $L^{2}$ through $z$. Any two flat $L_{1}^{2}$ containing $N$ must intersect $L^{2}$ in a line through $z$.

The plane $L_{1}^{2}$ cannot be a doubly timelike because of Lemma 4.2 and the fact that $K$ has only one component. Therefore, $L_{1}^{2}$ is neutral and contains two lines on $K$ parallel to $N$. It follows $K$ must be a cylinder with generators parallel to $N$.

Projecting $R$ onto $L^{2}$ along parallels to $N$ gives $R=R^{2} \times N^{1}$ for $R^{2}$ the spacelike plane $L^{2}$.

If $K$ has two components $K_{1}$ and $K_{2}$ in dimension three, then $K_{1}=$ $-K_{2}$ since otherwise there would be a spacelike plane $L^{2}$ through $z$ intersecting only one component of $K$ yet separating $A^{3}$. Both $K_{1}$ and $K_{2}$ must be flat since if $x, y \in K_{1}$ with $x \neq y$, the two flat $L_{1}^{2}$ containing $x, y$ and $z$ would have to be neutral.

It can easily be shown that for $K$ having two components, the space is always topologically isometric to $\left(x_{1}, x_{2}, x_{3}\right)$-space with the distance from $\left(a_{1}, a_{2}, a_{3}\right)$ to $\left(b_{1}, b_{2}, b_{3}\right)$ given by $\left|a_{1}-b_{1}\right| . K$ consists of two parallel planes and $R=R^{1} \times N^{2}$ for $R^{1}$ the real line.

6. Higher dimensional spaces. The $n$ dimensional situation is now investigated by the use of $r$-flats.

Lemma 6.1. $K_{1}, K_{2}, K_{3}$ be three distinct components of $K$, then two are reflections through $z$ of each other.

Proof. Consider $p_{i} \in K_{i}$ for $i=1,2,3$ and let $L^{3}$ be a three flat containing $z, p_{1}, p_{2}$, and $p_{3}$. Let $S_{i}=K_{i} \cap L^{3}$, then $S_{1}, S_{2}$, and $S_{3}$ are disjoint components of $K \cap L^{3}$. By the last section $K \cap L^{3}$ has either three or four components, and in any case, any three of the components 
of $K \cap L^{3}$ contain a pair that are symmetric to each other. If we assume $S_{1}=-S_{2}$ then clearly $K_{1}=-K_{2}$.

LEMMA 6.2. $K$ has at most four components. If $K$ does have four components $K_{1}, K_{2}, K_{3}$ and $K_{4}$, without loss of generality, one may assume $K_{1}=-K_{3}$ and $K_{2}=-K_{4}$.

Proof. Assume $K$ has five components $K_{1}, K_{2}, K_{3}, K_{4}$ and $K_{5}$. Then lemma 6.1 applied to $K_{1}, K_{2}$ and $K_{3}$ allows the assumption $K_{3}=-K_{1}$. Applying Lemma 6.1 to $K_{1}, K_{2}$ and $K_{4}$ yields $K_{2}=-K_{4}$.

Let $p_{1} \in K_{1}, p_{2} \in K_{2}$ and $p_{5} \in K_{5}$, then let $L_{3}$ be a three flat containing $p_{1}, p_{2}, p_{5}$ and $z$. $K \cap L^{3}$ then contains five disjoint components, which is impossible by Lemma 5.3 .

Lemma 6.3. Let $N_{x} \subset K$ then if one of the following holds, $N_{z}$ is a line of reduction.

(1) $K$ has exactly one component.

(2) $K$ has exactly two components $K_{1}$ and $K_{2}$ that are symmetric to each other.

(3) $K$ has exactly three components $K_{1}, K_{2}, K_{3}$ with $K_{3}=-K_{3}$ and $N_{x} \subset K_{1} \cup K_{2}$.

(4) $K$ has four components.

Proof. The proofs of the above four cases all follow the same general pattern. Therefore, the first case is the only one discussed.

If $N_{x} \subset K$ and $K$ has one component, consider $y \in R$ and let $L^{3}$ be a three flat containing $z, y$ and $N_{x}$. Either $N_{y} \subset K$ or else $K \cap L^{3}$ has three components. If $K \cap L^{3}$ has three components, there is a two flat $L^{2} \subset L^{3}$ through $z$ that is doubly timelike. But then $K \cap L^{2}$ has four components, and Lemma 4.2 would imply $K$ had more than one component.

For convenience the following notation is adopted. If $k, p, \cdots, m$ are $r$ distinct integers from the set $1,2, \cdots, n$ let $L_{k p \cdots m}^{r}$ be the unique $r^{r}$-flat through the $x_{k}, x_{p}, \cdots, x_{m}$ axes. If $L_{0}$ is a line with $L_{0} \not \subset L_{k p \cdots m}^{r}$ let $L_{v i p \cdots m}^{r+1}$ be the $r+1$ flat containing $L_{0}$ and $L_{k p \cdots m}^{r}$. Here we assume $L_{0} \cap L_{k p \cdots m}^{r} \neq \phi$.

Repeated application of the last lemma gives the following partial description of the nonreducible spaces:

THEOREM 6.4. In all cases $K$ has at most four components. Let $R$ be nonreducible.

(1) If $K$ has one component, then $R$ is a Minkowski G-space.

(2) If $K$ has two components that are opposed to each other then. $R$ is isometric to the real line.

(3) If $K$ has three components, then one is symmetric about $z$ 
and the other two are strictly convex.

(4) If $K$ has four components, then $R$ is a doubly timelike plane.

The case where $K$ has two components which are not opposed is discussed in Theorem 6.13 and additional information on the case of three components is found in Theorem 6.8.

Lemma 6.7. Let $n=3$ and $K$ have three components. Assume coordinates $x_{1}, x_{2}, x_{3}$ are chosen such that the light cone is given by $x_{1}^{2}+x_{2}^{2}=x_{3}^{2}$. Then the plane $x_{3}=0$ intersects $K_{3}$ in a set $x_{1}^{2}+x_{2}^{2}=a^{2}$ for some $a>0$.

Proof. Let $p$ lie on $K_{3}$ and in the plane $x_{3}=0$. For some $a>0$ the point $p$ lies on $x_{1}^{2}+x_{2}^{2}-x_{3}^{2}=a^{2}$. We claim that the only hyperboloid of one sheet containing $p$ that has $C$ as light cone is $x_{1}^{2}+x_{2}^{2}-x_{3}^{2}=a^{2}$.

Since $p$ is contained in exactly two planes tangent to $C$, the two lines on $K_{3}$ through $p$ are determined. For any $q$ on one of these two lines, the same argument yields that the two lines on $K_{3}$ through $q$ are determined. It follows $K_{3}$ is determined by $p$ and $C$.

Consider now $n>3$ and extend $A^{n}$ to $P^{n}$ by adding a hyperplane $L_{\infty}^{n-1}$ at $\infty$. Let the projective lines that contain the lines of the light cone $C$ intersect $L_{\infty}^{n-1}$ in a set $C_{\infty}$.

If $R$ is nonreducible and $K$ has three components, let $L_{0}^{n-1}$ be a supporting hyperplane to $K_{1}$. If $L^{n-1}$ is the hyperplane parallel to $L_{0}^{n-1}$ through $z$, then $L^{n-1} \cap C=z$. Otherwize $L^{n-1} \cap C$ would contain a line $N$. For $p \in L_{0}^{n-1} \cap K_{1}$ then the two flat $L^{2}$ through $p$ and $N$ would be neutral or doubly timelike. It could not be neutral because of Lemma 6.3. It could not be doubly timelike since then $N_{p}$ would not be a supporting line of $K_{1}$.

Set $L^{n-1} \cap L_{\infty}^{n-1}=L_{\infty}^{n-2}$ an $n-2$ dimensional flat. By taking $L_{\infty}^{n-2}$ as the $n-2$ flat at $\infty$ of $L_{\infty}^{n-1}$ the set $L_{\infty}^{n-1}-L_{\infty}^{n-2}$ becomes an $n-1$ dimensional affine space. Let $x, y \in C_{\infty}$ for $x \neq y$ and let $L_{1}^{2}$ be the two flat containing $x, y$ and $z$. Then $L_{1}^{2} \cap A^{n}$ is a doubly timelike plane. In the same manner as the argument after Lemma 5.1, we conclude $C_{\infty}$ is a strictly convex $n-2$ dimensional surface in the space $L_{\infty}^{n-1}-L_{\infty}^{n-2}$.

Lemma 6.6. $\quad C_{\infty}$ is an ellipsoid in $L_{\infty}^{n-1}-L_{\infty}^{n-2}$.

Proof. Let $L_{\infty}^{2}$ be a two flat in $L_{\infty}^{n-1}$ with $L_{\infty}^{2} \cap C_{\infty}$ containing more than one point. Let $L^{3}$ be the three flat containing $z$ and $L_{\infty}^{2}$. Then $L^{3} \cap A^{n}$ is an indefinite metric space whose unit sphere has three components. By Theorem 5.2, $L_{\infty}^{2} \cap C_{\infty}$ is an ellipse and hence by [2, p. 91] $C_{\infty}$ is an ellipsoid.

Take now coordinates $x_{1}, x_{2}, \cdots, x_{n}$ in $A^{n}$ such that $C$ has the form 
$x_{n}^{2}=x_{1}^{2}+\cdots+x_{n-1}^{2}$ and let $L_{1}^{n-1}$ be the hyperplane $x_{n}=0$.

LemmA 6.7. $L_{1}^{n-1} \cap K$ has the form $x_{1}^{2}+\cdots+x_{n-1}^{2}=a^{2}$ for $a>0$.

Proof. Let $L^{2}$ be any two flat in $L_{1}^{n-1}$ passing through $z$. Let $L^{3}$ be the three flat containing $L^{2}$ and the $x_{n}$ axis. Since $L^{3} \cap K$ always has three components, $L^{2} \cap K$ is always an ellipse of center $z$. Therefore, $L_{1}^{n-1} \cap K$ is an ellipsoid in $L_{1}^{n-1}$ of center $z$.

If $L^{2}$ contains the $x_{i}$ and $x_{j}$ axis Lemma 6.5 implies $L^{2} \cap K_{3}$ has the form $x_{i}^{2}+x_{j}^{2}=a_{i j}^{2}$. If $p_{i}$ and $p_{j}$ are points of $L^{2} \cap K_{3}$ that lie on the $i^{\text {th }}$ and $j^{\text {th }}$ axes respectively, $\left|p_{i}\right|^{2}=\left|p_{j}\right|^{2}=a_{1 j}^{2}$. Therefore, $a_{i j}$ is independent of $i$ and $j$. Setting $a=a_{i j}$ yields the desired result.

THEOREM 6.8. Let $R$ be nonreducible and $K$ have three components. If $K_{3}$ is the components of $K$ symmetric about $z$ it is a quadric. In proper affine coordinates $K_{3}$ is given by

$$
x_{1}^{2}+\cdots+x_{n-1}^{2}-x_{n}^{2}=a^{2} .
$$

Proof. Using the same notation as in Lemma 6.9 define

$$
S=\left\{\left(x_{1}, x_{2}, \cdots, x_{n}\right) \mid x_{1}^{2}+\cdots+x_{n-1}^{2}-x_{n}^{2}=a^{2}\right\} .
$$

If $L^{3}$ contains the $x_{n}$ axis then $L^{3} \cap S=L^{3} \cap K_{3}$. The result follows by letting $L^{3}$ sweep out $A^{n}$.

In order to investigate nonreducible spaces in which $K$ has two components, we first consider nondegenerate central quadrics that have $z$ as a center. The general form in affine space is

$$
\sum_{i, j=1}^{n} a_{i j} x_{i} x_{j}=1 \text { where } a_{i j}=a_{j i} \text { and } \operatorname{det}\left(a_{i j}\right) \neq 0 .
$$

If two such quadrics $E_{1}$ and $E_{2}$ are given respectively by

$$
\sum a_{i j} x_{i} x_{j}=1 \text { and } \sum a_{i j} x_{i} x_{j}=-\lambda^{2} \text { for } \lambda>0,
$$

they will be called semiconjugate. We will refer to $E_{1}$ as the $\lambda$ semiconjugate to $E_{2}$. For $\lambda=1$ the quadrics are conjugate in the usual sense. Notice that one of the quadrics does not have a real locus if the quadric form is definite.

LEMMA 6.9. Suppose the nonempty sets $B_{1}$ and $B_{2}$ contained in $\bigcup_{i \neq j} L_{i j}^{2}$ are such that the locus $B_{2} \cap L_{i j}^{2}$ is always the $\lambda$ semiconjugate quadric to $B_{1} \cap L_{i j}^{2}$ for fixed $\lambda$. Then there are exactly two central quadrics $E_{1}$ and $E_{2}$ such that $E_{1} \cap L_{i j}^{2}=B_{1} \cap L_{i j}^{2}$ and $E_{2} \cap L_{i j}^{2}=$ $B_{2} \cap L_{i j}^{2}$ for all $i \neq j$. Furthermore, $E_{2}$ is the $\lambda$ semiconjugate to $E_{1}$.

Lemma 6.10. Let $n=4$ and $K$ have two components $K_{1}$ and $K_{2}$ 
each symmetric about $z$. Let $L^{3}$ be a three flat through $z$ such that $L^{3} \cap K$ has three components. Then $L^{3} \cap K$ consists of two semiconjugate quadrics.

Proof. By Theorem 5.2 one component of $L^{3} \cap K$ must be a hyperboloid of one sheet. Choose coordinates $x_{1}, x_{2}, x_{3}$ in $L^{3}$ such that $L^{3} \cap C$ takes the form $x_{1}^{2}+x_{2}^{2}=x_{3}^{2}$. Let $L^{3} \cap K$ have components $S_{1}, S_{2}, S_{3}$ with $S_{3}=-S_{3}$. For some $a>0, S_{3}$ is given by $x_{1}^{2}+x_{2}^{2}-x_{3}^{2}=a^{2}$. Let $L_{0}$ be a line through $z$ in $L_{12}^{2}$.

In $R$ let $L^{2}$ be a spacelike plane containing the $x_{3}$ axis, so $L^{2} \not \subset L^{3}$. Choose the $x_{4}$ axis in $L^{2}$. Assume $K$ has components $K_{1}$ and $K_{2}$ with $S_{3} \subset K_{1}$, then $L_{c 34}^{3} \cap K_{2}$ is a hyperboloid of one sheet in $L_{034}^{3}$. Consequently, $L_{03}^{2} \cap K_{2}$ is a hyperbola. This hyperbola is determined given only the intersection of $K_{2}$ with the $x_{3}$ axis and the intersection of $L_{03}^{2}$ with the surface $x_{1}^{2}+x_{2}^{2}=x_{3}^{2}$ in $L^{3}$.

Revolving $L_{0}$ in the plane $L_{12}^{2}$ shows $L^{3} \cap K_{2}$ consists of a hyperboloid of two sheets that is a semiconjugate of $L^{3} \cap K_{1}$.

LEMMA 6.11. If $n=4$ and $K$ has two symmetric components, they are semiconjugate quadrics.

Proof. Let the notation and coordinates be the same as in the last proof. Set $B_{1}=\bigcup_{i \neq j}\left(L_{i j}^{2} \cap K_{1}\right)$ and $B_{2}=\bigcup_{i \neq j}\left(L_{i j}^{2} \cap K_{2}\right)$.

If $L^{3} \cap K_{2}$ is the $\lambda$ semiconjugate to $L^{3} \cap K_{1}$ in $L^{3}$, then $L_{034}^{3} \cap K_{2}$ is the $\lambda$ semiconjugate to $L_{034}^{3} \cap K_{1}$ in $L_{034}^{3}$ for the same $\lambda$. This follows since $L_{03}^{2}$ is common to both three flats and intersects both components of $K$. Therefore, $B_{1}$ and $B_{2}$ satisfy the hypothesis of Lemma 6.9. Let $E_{1}$ and $E_{2}$ be the semiconjugate quadrics determined by $B_{1}$ and $B_{2}$.

$L^{3} \cap E_{1}=L^{3} \cap K_{1}$ since each are quadrics in $L^{3}$ determined by $B_{1} \cap L^{3}$ and $B_{2} \cap L^{3}$. By the same reasoning, $L^{3} \cap E_{2}=L^{3} \cap K_{2}$. Also $L_{124}^{3} \cap K_{i}=L_{124}^{3} \cap K_{i}$ for $i=1,2$.

Therefore, $L_{0 j}^{2} \cap K_{i}=L_{0 j}^{2} \cap E_{i}$ for $i=1,2$ and $j=3$, 4. But then using Lemma 6.11 one last time, we find $L_{034}^{3} \cap E_{i}=L_{034}^{3} \cap K_{i}$. By revolving $L_{0}$ in $L_{12}^{2}$ it follows $E_{i}=K_{i}$ for $i=1,2$.

Lemma 6.12. Let $n=5$ and $K$ have two components $K_{1}$ and $K_{2}$ symmetric about $z$. If $R$ is not reducible, $K_{1}$ and $K_{2}$ are semiconjugate quadrics.

Proof. Two cases are considered.

Case 1. Let there exist a three flat $L^{3}$ through $z$ such that $L^{3} \cap K$ has one component. Assume $L^{3} \cap K_{2} \neq \phi$. Choose coordinates $x_{1}, x_{2}, x_{3}$ in $L^{3}$. We may assume that $L_{12}^{2}, L_{13}^{2}, L_{23}^{2}$ are spacelike planes. Choose 
coordinates $x_{4}, x_{5}$ such that $L_{45}^{2}$ is spacelike and intersects $K_{1}$. By arguments as in Lemma 6.10 and Lemma 6.11, it is possible to show $L_{i j}^{2} \cap K_{1}$ and $L_{i j}^{2} \cap K_{2}$ are always semiconjugate quadrics for fixed $\lambda$. Therefore, $B_{1}=\bigcup_{i \neq j}\left(L_{i j}^{2} \cap K_{1}\right)$ and $B=\bigcup_{i \neq j}\left(L_{i j}^{2} \cap K_{2}\right)$ satisfy the hypothesis of Lemma 6.9.

Let $E_{1}$ and $E_{2}$ be the quadrics determined by $B_{1}$ and $B_{2}$. Let $L_{0}$ be a line through $z$ in $L_{12}^{2}$. Since $L_{12 j}^{2} \cap E_{i}=L_{12 j}^{3} \cap K_{i}$, clearly $L_{0 j}^{2} \cap E_{i}=$ $L_{0 j}^{2} \cap K_{1}$ for $i=1,2$ and $j=3,4,5$. Therefore $L_{0345}^{4} \cap E_{i}=L_{i 345}^{4} \cap K_{i}$. By revolving $L_{0}$ in $L_{12}^{2}$ it follows that $E_{i}=K_{i}$.

Case 2. Assume no $L^{3}$ through $z$ exists with $L^{3} \cap K$ having only one component. We will show this leads to a contradiction.

Choose coordinates $x_{1}, x_{2}, x_{3}, x_{4}, x_{5}$ such that $L_{12}^{2}$ and $L_{34}^{2}$ are spacelike planes intersecting respectively $K_{1}$ and $K_{2}$. By Theorem 6.8 , the set $K \cap L_{2345}^{4}$ cannot have exactly three components. Consequently, $L_{2345}^{3} \cap K$ consists of two symmetric components. The same must also be true of $L_{1235}^{4} \cap K$.

By Lemma 6.11 the sets $L_{1234}^{4} \cap K, L_{2345}^{4} \cap K$ and $L_{1235}^{4} \cap K$ each consists of two quadrics. In each of the three sets one quadric is the semiconjugate of the other for some fixed $\lambda$. Define

$$
B_{1}=\bigcup_{i \neq j}\left(L_{i j}^{2} \cap K_{1}\right) \text { and } B_{2}=\bigcup_{i \neq j}\left(L_{i j}^{2} \cap K_{2}\right) .
$$

Let $E_{1}$ and $E_{2}$ be the quadrics determined.

Let $L_{0}$ be a line through $z$ in $L_{12}^{2}$. Then $L_{0 j}^{2} \cap K_{i}=L_{0 j}^{2} \cap E_{i}$ for $j=3,4,5$ and $i=1,2$. Therefore, $L_{0345}^{4} \cap E_{i}=L_{0345}^{4} \cap K_{i}$ and revolving $L_{0}$ in $L_{12}^{2}$ gives $E_{i}=K_{i}$ for $i=1,2$.

Then in proper affine coordinates $y_{1}, y_{2}, y_{3}, y_{4}, y_{5}$ the components of $K$ are given by $y_{1}^{2}+y_{2}^{2}+y_{3}^{2}-y_{4}^{2}-y_{5}^{2}=1$ and $y_{1}^{2}+y_{2}^{2}+y_{3}^{2}-y_{4}^{2}-y_{5}^{2}=$ $-\lambda^{2}$. This contradicts the assumption of Case 2 .

The $n$ dimensional case now follows using induction.

TheOREM 6.13. If $R$ is not reducible and $K$ has two components which are not opposed, then $n \geqq 4$ and the components are semiconjugate quadrics.

Proof. Assume $n \geqq 6$. Take $L^{n-1}$ to be a hyperplane containing $L_{1}^{2}$ and $L_{2}^{2}$, which are spacelike two flats through $z$ with $L_{i}^{2} \cap K_{i} \neq \phi$. Then $L^{n-1} \cap K$ has exactly two symmetric components. Because of Lemma 6.12 , there exists an $L^{3}$ through $z$ and contained in $L^{n-1}$ with $L^{3} \cap K$ having one component. Take the $x_{1}, x_{2}, x_{3}$ affine coordinates in $L^{3}$ and $x_{1}, x_{2}, \cdots, x_{n-1}$ affine coordinates in $L^{n-1}$. For $p \in K-L^{n-1}$ let the $x_{n}$ axis be $L(z, p)$. Take $L_{0}$ to be a line through $z$ in $L_{12}^{2}$. By 
induction $L_{034 \omega_{n} \cdots n}^{n-1} \cap K_{i}$ must consist of two semiconjugate quadrics. The argument is the same as before, letting $L_{0}$ revolve in $L_{12}^{2}$.

An interesting result of this section is the following.

CoRollary 6.14. If $R$ is a nonreducible Minkowski space and not a $G$-space, then any spacelike plane in $R$ is euclidean.

\section{REFERENCES}

1. J. K. Beem and P. Y. Woo, Doubly timelike surfaces, Amer. Math. Soc. Memoir 92, 1970.

2. H. Busemann, The geometry of geodesics, Academic Press, New York, 1955.

3. - Timelike spaces, Dissertationes Mathematicae, Warszawa 53 (1967).

4. H. Busemann and J. K. Beem, Axioms for indefinite metrics, Circolo Matematico di Palermo (1966), 223-246.

5. H. Busemann and P. J. Kelly, Projective geometry and projective metrics, Academic Press, New York, 1953.

Received August 25, 1969. This paper is part of a $\mathrm{Ph}$. D. dissertation written under the direction of Professor H. Busemann at the University of Southern California.

UNIVERSITY OF MISSOURI

Columbia, Missouri 



\section{PACIFIC JOURNAL OF MATHEMATICS}

\section{EDITORS}

H. SAMELSON

Stanford University

Stanford, California 94305

\section{RichaRd PIERCe}

University of Washington

Seattle, Washington 98105
J. DUGUNDJI

Department of Mathematics

University of Southern California

Los Angeles, California 90007

RICHARD ARENS

University of California

Los Angeles, California 90024

\section{ASSOCIATE EDITORS}

E. F. BECKENBACH

B. H. NeUManN

F. WOLF

K. YosHIDA

\section{SUPPORTING INSTITUTIONS}

UNIVERSITY OF BRITISH COLUMBIA CALIFORNIA INSTITUTE OF TECHNOLOGY UNIVERSITY OF CALIFORNIA MONTANA STATE UNIVERSITY

UNIVERSITY OF NEVADA

NEW MEXICO STATE UNIVERSITY

OREGON STATE UNIVERSITY

UNIVERSITY OF OREGON

OSAKA UNIVERSITY

UNIVERSITY OF SOUTHERN CALIFORNIA
STANFORD UNIVERSITY

UNIVERSITY OF TOKYO

UNIVERSITY OF UTAH

WASHINGTON STATE UNIVERSITY

UNIVERSITY OF WASHINGTON

${ }^{*} \quad{ }^{*} \quad{ }^{*}$
AMERICAN MATHEMATICAL SOCIETY
CHEVRON RESEARCH CORPORATION
TRW SYSTEMS
NAVAL WEAPONS CENTER




\section{Pacific Journal of Mathematics}

\section{Vol. 33, No. $1 \quad$ March, 1970}

Mir Maswood Ali, On some extremal simplexes ................... 1

Silvio Aurora, On normed rings with monotone multiplication........... 15

Silvio Aurora, Normed fields which extend normed rings of integers....... 21

John Kelly Beem, Indefinite Minkowski spaces..................... 29

T. F. Bridgland, Trajectory integrals of set valued functions ........... 43

Robert Jay Buck, A generalized Hausdorff dimension for functions and sets ......................................... 69

Vlastimil B. Dlab, A characterization of perfect rings . . . . . . . . . . . . 79

Edward Richard Fadell, Some examples in fixed point theory ............ 89

Michael Benton Freeman, Tangential Cauchy-Riemann equations and uniform approximation ............................. 101

Barry J. Gardner, Torsion classes and pure subgroups ................ 109

Vinod B. Goyal, Bounds for the solution of a certain class of nonlinear

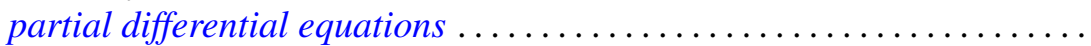

Fu Cheng Hsiang, On C, 1 summability factors of Fourier series at a given

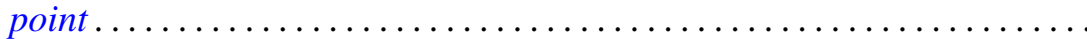

Lawrence Stanislaus Husch, Jr., Homotopy groups of PL-embedding

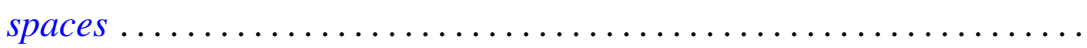

Daniel Ralph Lewis, Integration with respect to vector measures..........

Marion-Josephine Lim, $\mathscr{L}-2$ subspaces of Grassmann product spaces

Stephen J. Pierce, Orthogonal groups of positive definite multilinear functionals

W. J. Pugh and S. M. Shah, On the growth of entire functions of bounded index.

Siddani Bhaskara Rao and Ayyagari Ramachandra Rao, Existence of triconnected graphs with prescribed degrees . . .

Ralph Tyrrell Rockafellar, On the maximal monotonicity of subdifferential

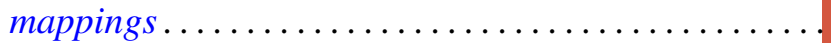

R. Shantaram, Convergence of a sequence of transformations of distribution functions. II ...............................

Julianne Souchek, Rings of analytic functions..............

Ted Joe Suffridge, The principle of subordination applied to functions of several variables...

Wei-lung Ting, On secondary characteristic classes in cobordism

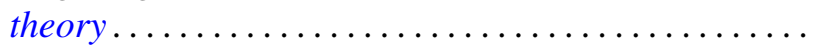

Pak-Ken Wong, Continuous complementors on $B^{*}$-algebras ...

Miyuki Yamada, On a regular semigroup in which the idempotents form a band. 